Military Technical College Kobry El-Kobba Cairo, Egypt

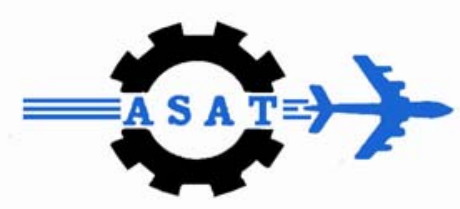

12-th International Conference on

Aerospace Sciences \& Aviation Technology

\title{
SOLUTION OF ECONOMIC DISPATCH PROBLEM USING EVOLUTIONARY PROGRAMMING TECHNIQUES
}

\author{
F. M. Bendary*
}

A. A. Ishak*

M. I. Mosaad**

\section{ABSTRACT}

This paper presents a comparison between various methods used to solve economic dispatch (ED) problem. These methods are heuristic methods such as Genetic Algorithms (GAs) and Particle Swarm Optimization (PSO) algorithm. Comparisons between these heuristic methods and conventional methods such as Interior Point method (IPM) are introduced. The objective function that consists of the fuel (generation) cost is minimized. Numerical examples typical to each method are presented. The solutions method adopted has yielded encouraging results that proved useful in the improvement of economic dispatch environment.

\section{KEY WORDS}

Economic dispatch, genetic algorithms, particle swarm optimization, and interior point method

\footnotetext{
*Fahmybendary10@yahoo.com

**m_i_mosaad@hotmail.com
} 


\section{INTRODUCTION}

Electric power systems have experienced continuous growth in its entire main constituencies sectors namely, generation, transmission, and distribution. In the past, transmission systems were characterized by a low order of interconnection; hence, it was less complicated in allocating the share of the load among several generating units [1]. The increase in load sizes and operational of complexity brought about by widespread interconnection of transmission systems, some encompassing continental distances, introduced major difficulties to the electrical power networks operation. It became necessary for many electrical utilities to operate in close proximity to their system ultimate operating capacity. It became impractical to determine appropriate operating strategies mainly based on the observations and the experiences of the operator.

The operating strategy had to be revised, and new concepts based on economic considerations were adopted. Economic dispatch (ED) problem has become one of the fundamental issues in power system operation. In essence, it is an optimization problem with the objective to reduce the total generation cost of units, while observing system constraints.

Optimal power flow (OPF) solution methods have been developed over the years to meet this very practical requirement of power system operation [2-5]. The optimal power flow problem; has been discussed since its introduction by Carpentier [6]. Because the OPF is a very large, non-linear mathematical programming problem, it has taken decades to develop efficient algorithms for its solution. Many different mathematical techniques have been employed for its solution. The majority of the techniques discussed in the literature use one of the following five methods, Lambda iteration method, Gradient method, Newton's method, Linear programming method and Interior point method [7-10].

Recently stochastic search techniques such as simulated annealing (SA), GAs, PSO and Artificial Neural Network (ANN) were introduced to allocate global or near global optimal solution. Although these methods have been employed to solve the complex nonlinear ED problem, they do not always guarantee the globally optimal solution, but they provide a reasonable solution in a short computation time.

It wasn't 1995 that streifferd [11] proposed the use of network flow algorithm to solve multiple area ED with tie line constrains. In 1998 the adaptive hopfield neural network were applied to solve the economic dispatch problem with piecewise quadratic cost function [12]. GAs with fuzzy logic controllers dedicated to adjust its crossover and mutation probabilities to solve combined environmental economic dispatch has been successfully applied [13]. This paper presents different methods to handle the ED problem using GAs and PSO algorithm from the heuristic methods, IPM from the classical methods and the comparison between them will also be presented. 


\section{PROBLEM FORMULATION}

The objective function $F$ is to determine the generation levels and the interchange power between each generating unit while satisfying a set of constrains as:

$$
\operatorname{Min}(\mathrm{F})=\operatorname{Min} \sum_{n=1}^{N} f_{n}\left(P_{n}\right)
$$

Where $f_{n}\left(P_{n}\right)$ is the fuel cost (operation cost) of unit $\mathrm{n}$, in terms of active power generated by this unit, $P_{n}$ and $N$ is the number of generators in the system. The cost function of the fuel cost has been approximated by the quadratic function as:

$$
f_{n}\left(P_{n}\right)=a_{n} P_{n}^{2}+b_{n} P_{n}+c_{n}
$$

Where $a_{n}, b_{n}$, and $c_{n}$ denotes the fuel cost coefficients. Subjected to the following constrains:

i- Power balance constraint

$$
\sum_{n=1}^{N} f_{n}\left(P_{n}\right)=P_{D}+\sum_{k=1}^{K} P_{l k}
$$

where $P_{l k}$ are the transmission line losses, $P_{D}$ is the demand power, and $\mathrm{k}$ is the total number of the lines in the system.

ii- Generation active power constraint

$$
P_{n_{\min }} \leq P_{n} \leq P_{n_{\max }}
$$

iii- Generation reactive power constraint

$$
Q_{n_{\min }} \leq Q_{n} \leq Q_{n_{\max }}
$$

where $Q_{n}$ is the reactive power at each generation unit $n$.

iv- Line limits constraint

$$
I_{k \min } \leq I_{k} \leq I_{k \max }
$$

where $I_{k}$ is the current flowing in line $k$.

$\mathrm{v}$ - The inequality constraint of the voltage $\mathrm{V}$ at each bus will read:

$V_{\min } \leq V \leq V_{\max }$ 


\section{OPTIMIZATION ALGORITHMS}

OPF is a tool used for both planning and operation of power systems. There exists various methods to solve the OPF problem. Heuristic methods may be used to solve combinatorial optimization problems. These methods are called "intelligent," because they move from one solution to another using rules close to the human reasoning. The heuristic algorithms will search for a solution inside a subspace of the total search space, they can provide in providing a good solution to a given problem in a reasonable computation time on one hand, but they do not assure the convergence to a global optimum solution. a paramount advantage of heuristic methods lies in the fact that they are not limited by restrictive assumptions about the search space like continuity, existence of derivative of objective function, etc... .

The existing heuristic methods exist; among them, we quote Tabu Search method (TS) [14], Simulated Annealing (SA) [15], Genetic Algorithms (GAs) [16], and Particle Swarm Optimization (PSO) algorithms [17]. Each method has its own merits and drawbacks. The TS is basically a deterministic method, and experience has shown that no random process might restrict the search in the set of solutions. The SA requires longer computational time. Further, there exist a number of important parameters that may prove to be difficult to determine, such as the cooling schedule.

In this research GAs and PSO algorithm are used to achieve OPF solution of power system.

\subsection{Genetic Algorithms}

Based on the mechanisms of natural selection and genetics, GAs are global search techniques dedicated to provide several possible solutions simultaneously and do not require any prior knowledge or special properties of the objective function [18]. Moreover, they always produce high quality solutions and, accordingly, are excellent methods for seeking optimal solution to complex problems. (GAs) starts with random generation of initial population which represent possible solutions of the problem; Then the fitness of each individual is evaluated and new populations are generated by genetic operators (Reproduction, Crossover and Mutation) until the maximal number of generation is reached. Figure.1 depicts the outline of (GAs) for optimization problems. The (GAs) differs from other optimization and search Procedures in four ways [19]:

1- GAs work with a coding of the parameter set, and not with the individual parameters. Therefore (GAs) can easily handle the integer or discrete variables.

2- GAs will search among a population of points, and not from a single point. Therefore (GAs) can provide a globally optimal solution.

3- GAs make of use only objective function information, and not their derivatives or other auxiliary knowledge, Therefore (GAs) can deal with the non-smooth, noncontinuous and non-differentiable functions which are actually exists in a practical optimization problem.

4- Finally GAs adopted probabilistic transition rules and not deterministic rules. 


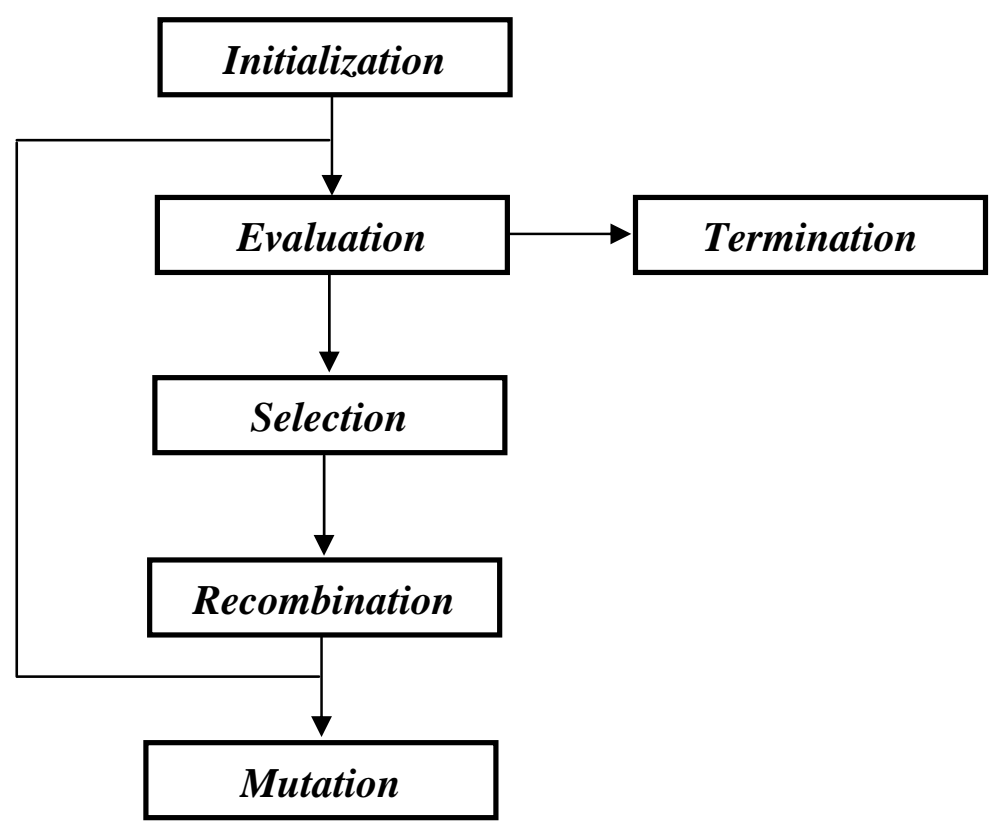

Fig. 1. Outline of GAs for optimization problems

\subsection{Particle Swarm Optimization Techniques}

In 1995, Kennedy and Eberhart first introduced the PSO algorithm [20], motivated by social behavior of organisms such as fish schooling and bird flocking. PSO, as an optimization tool, provides a population-based search procedure in which individuals called particles change their positions (states) with time. The basic assumption behind the PSO algorithm is birds find food by flocking and not individually. This leads to the assumption that information is owned jointly during in flocking. Basically PSO was developed for two-dimension solution space [20].

Let $\mathrm{x}$ and $\mathrm{y}$ denote a particle coordinates (position) and its corresponding flight speed (velocity) $V_{x}$ and $V_{y}$ in both the $x$ and $y$ directions. Modification of the individual position is realized through the velocity and position information.

The PSO algorithm for a $\mathrm{N}$-dimensional problem formulation can be implemented as follows. Let $P$ be the particle position and $V$ is the velocity in a search space. Consider $i$ as a particle in the total population (swarm). The $i^{\text {th }}$ particle position can be represented by $\mathrm{Pi}=\left(\mathrm{P}_{\mathrm{i} 1}, \mathrm{P}_{\mathrm{i} 2}, \mathrm{P}_{\mathrm{i} 3} \ldots \mathrm{P}_{\mathrm{iN}}\right)$ in the $\mathrm{N}$-dimensional space. The best previous position of the $i^{\text {th }}$ particle is recorded and represented as Pbesti= $\left(\right.$ Pbest $_{11}$, Pbest $_{i 2}$, Pbest $\mathrm{i}_{33}$, Pbest $\left._{i j}\right)$. The index of the best particle among all the particles in the group is represented by gbest. The velocity $i^{\text {th }}$ particle is represented as $\mathrm{Vi}=\left(\mathrm{V}_{\mathrm{i} 1}, \mathrm{~V}_{\mathrm{i} 2}, \mathrm{~V}_{\mathrm{i} 3} \ldots \mathrm{V}_{\mathrm{ij}}\right)$. The modified velocity and position of each particle can be calculated using the current velocity and the distance from Pbest to gbest as indicated in the following formulae:

$V_{i j}^{(t+1)}=w * V_{i j}^{(t)}+c_{1} *$ rand $_{1} *\left(\right.$ Pbest $_{i j}-$ Pbest $\left._{i j}^{(t)}\right)+c_{2} *$ rand $_{2} *\left(\right.$ gbest $_{i}-$ Pbest $\left._{i j}^{(t)}\right)$ 


$$
\begin{aligned}
& p_{i j}^{(t+1)}=p_{i j}^{(t)}+v_{i j}^{(t+1)} \\
& i=1,2, \ldots \ldots . . I \quad \text { and } \quad j=1,2, \ldots \ldots . . . N \text {, where: }
\end{aligned}
$$

$\mathrm{N} \quad$ Denotes number of dimensions in a particle

I Denotes number of particles

w Denotes inertia weight factor

$\mathrm{t} \quad$ Denotes pointer of iterations

$\mathrm{C}_{1}$ and $\mathrm{c}_{2} \quad$ Denotes accelerating constant

rand $_{1}$ and rand 2 Denotes uniform random value in the range of $[0,1]$

$v_{i j}^{(t)}$

$p_{i j}^{(t)}$

Denotes velocity of the jth dimension in the ith particle

Denotes current position of the jth dimension in the ith particle at iteration $\mathrm{t}$

Inertia weighting factor $w$ has provided improved performance when using the linearly decreasing value [17]. Its value is linearly decreasing from about 0.9 to 0.4 during a run. Suitable selection of $\mathrm{w}$ provides a balance between global and local exploration and exploitation, and results in less iteration on average to find a sufficiently optimal solution. Its value is set according to the following equation:

$w=w_{\max }-\frac{w_{\max }-w_{\min }}{t_{\max }} * t$

Where $\quad w_{\max }$ and $w_{\min }$ are both random numbers called initial and final Weights respectively.

$t_{\max } \quad$ maximum iteration number

$\mathrm{t}$ the current iteration number

In equation (8), the first term indicates the current velocity of the particle, the second term represents the cognitive part of PSO where the particle changes its velocity based on its own thinking and memory, and finally the third term represents the social part of PSO where the particle changes its velocity based on the social-psychological adaptation of knowledge [20].

\section{DEVELOPMENT OF THE PROPOSED METHODS}

In this paper, the process of determining the generation levels and the interchange power between each generating unit in order to minimize the overall generating cost using GAs and PSO are developed to obtain efficiently a high quality solution within practical power system operation. 


\subsection{Implementation of GAs in ED Problem}

GA is essentially a method to generate a new population or generation from a given population. In this process the selection, crossover, and mutation operators are being used. Each member of the population, called a chromosome, is a possible solution for the problem under consideration, and is represented as a binary chain.

Members of each generation are ranked according to a specific criterion called fitness. The choice operator gives those members with a higher fitness ranking a better chance of being present in the next generation. The crossover and mutation operators are applied to each chromosome with a specific probability and cause new chromosomes to be present in the new generation.

To solve ED problem using GAs, first of all the possible solutions must be coded in chromosomes that is the active power generated by unit $\mathrm{n}$, Pn. Series binary coding is used in this paper. Next a fitness function to compare the chromosomes has to be defined. The value of the fitness function is equal to the value of the cost function in equation (1), which has to be minimized. Therefore to calculate the fitness of a chromosome, the fuel cost of the units has to be calculated using the information in the chromosome.

\subsection{Implementation of PSO Algorithm in ED Problem}

In this paper the solution to ED using PSO algorithm is introduced. The PSO algorithm is mainly used to determine the optimal generation power of each unit, for the purpose of minimizing the total generation cost. Its implementation encompasses the following six steps:

Step 1 The number of generating units will specify the dimension. The particles are randomly generated between the maximum and minimum limits of the generators. If there are $\mathrm{N}$ units, the $\mathrm{i}^{\text {th }}$ particle is represented as follows: $\mathrm{Pi}=\left(\mathrm{P}_{\mathrm{i} 1}, \mathrm{P}_{\mathrm{i} 2}, \mathrm{P}_{\mathrm{i} 3} \ldots \mathrm{P}_{\mathrm{iN}}\right)$.

Step 2 The particles velocities are generated randomly in the range of $\left[-V_{j}^{\max }, V_{j}{ }^{\max }\right]$. The maximum velocity limit was set at $10-20 \%$ of the dynamic range of the variables on each dimension [17].

Step 3 The objective function values of the particles are evaluated using equation (1). The computed values are set as Pbest value of the particles.

Step 4 The best value among all the Pbest values is identified and denoted as gbest.

Step 5 New velocities for all the dimensions in each particle are calculated using equation (8). Then the position of each particle is updated using equation (9).

Step 6 The objective function values are calculated for the updated positions of the particles. If the new value is better than the previous Pbest, the new value is set to be Pbest. When the limiting criteria are met, the positions of particles represented by gbest will provide the optimal solution, otherwise the procedure is repeated from step 4. 


\section{5- CASE STUDY AND RESULTS}

To validate the effectiveness of the suggested methods used in solving the ED problem, an appropriate study case was selected and solved using the conventional method (IPM), the (GAs) and (PSO) methods.

The case study, is based on the IEEE 14 bus system comprising three generating units [21], eleven loads and sixteen lines with a system total load of the system is $260.89 \mathrm{MW}$ .The cost coefficients of the generating units are tabulated in Table (1). In these proposed methods, Newton-Raphson method was used for power flow. The solution was obtained using the MATLAB software package.

Table 1. Fuel cost coefficients of the IEEE 14 bus system

\begin{tabular}{|c|c|c|c|}
\hline Generator & $\mathrm{a}$ & $\mathrm{b}$ & $\mathrm{c}$ \\
\hline 1 & 0.0050 & 1.89 & 150 \\
\hline 2 & 0.0055 & 2.00 & 115 \\
\hline 3 & 0.0060 & 3.5 & 40 \\
\hline
\end{tabular}

For implementing the GA in ED problem, population size of 100 was taken and the maximum number of generations was taken as 100 . In the PSO method the population size of 100 was taken and the maximum number of generations was taken as 100 . The inertia weight factor is set by (10), where $w_{\max }$ and $w_{\min }$ are 0.9 and 0.2 respectively.

The acceleration constants $\mathrm{c}_{1}$ and $\mathrm{c}_{2}$ were all made equal to 2 .

The solution to the system thus formed was performed using various optimization methods, namely, the conventional (IPM) method and the suggested (GAs) and (PSO) methods. The results of this case study are presented in Table (2).

The results of the suggested methods are shown to be close with those obtained from the classical approach (IPM). Moreover the careful study of these results shows savings of the order 10.4 U.S.\$ /hr using the (GAs) method and 17.5 U.S.\$ /hr using the (PSO) in favor of the methods suggested in this paper.

Table 2. Simulation results of ED with IPM, GAs and PSO for IEEE 14 bus system

\begin{tabular}{|c|c|c|c|}
\hline Generating Unit & IPM & GAs & PSO \\
\cline { 2 - 4 } & $\mathrm{Pg}(\mathrm{MW})$ & $\mathrm{Pg}(\mathrm{MW})$ & $\mathrm{Pg}(\mathrm{MW})$ \\
\hline G1 & 192.32 & 185.63 & 186.34 \\
\hline G2 & 38.2 & 41.2 & 39.67 \\
\hline G3 & 41.9 & 43.78 & 42.28 \\
\hline Fuel Cost $\$$ /hr & 1095 & 1084.6 & 1077.5 \\
\hline
\end{tabular}




\section{CONCLUSIONS}

In this paper, the (GAs) and (PSO) methods were successfully used to solve the ED problem with the power flow constraints and fuel cost function being approximated to a quadratic function. Results showed that (GAs) and (PSO) methods are well suited for obtaining the best solution for fuel cost functions of differentiable, nonsmooth, and nondifferentiable test system. The outcome of applying these methods to the system under test has yielded appreciable savings to the cost of the total generated power.

\section{REFERENCES}

[1] Alsac, O., Bright, J., Prais, M., Stott, B., 1990, "Further Developments in LP-based Optimal Power Flow “, IEEE Trans. Power Systems 5(3), pp.697-711

[2] Acha, E., Ambriz-Pe'rez, H., Fuerte-Esquivel, C.R., 2000, 'Advanced Transformer Control Modelling in an Optimal Power Flow Using Newton's Method', IEEE Trans. Power Systems 15(1), pp.290-298.

[3] Huneault, M., Galiana, F.D., 1991, 'A Survey of the Optimal Power Flow Literature', IEEE Trans. Power Systems 6(2), pp.762-770

[4] Monticelli, A., Liu,W.H.E., 1992, "Adaptive Movement Penalty Method for the Newton Optimal Power Flow", IEEE Trans. Power Systems 7(1),pp. 334-342.

[5] El-Hawary, M.E., Tsang, D.H., 1986, 'The Hydro-thermal Optimal Power Flow, A Practical Formulation and Solution Technique using Newton's Approach", IEEE Trans. Power Systems PWRS-1(3) ,pp157-167.

[6] J. Carpienter, "Contribution e l'étude do Dispatching Economique," Bulletin Society Française Electriciens, Vol. 3, August 1962.

[7] A. J. Wood and B. F. Wollenberg, Power Generation Operation and Control, New York, NY: John Wiley \& Sons, Inc., 1996, pp. 39-517.

[8] H. W. Dommel and W. F. Tinney, "Optimal Power Flow Solutions," IEEE Transactions on Power Apparatus and Systems, Vol. PAS-87, October 1968, pp. 1866-1876.

[9] D. I. Sun, B. Ashley, B. Brewer, A. Hughes and W. F. Tinney, "Optimal Power Flow by Newton Approach," IEEE Transactions on Power Apparatus and Systems, Vol. PAS-103, October 1984, pp. 2864-2880.

[10] O. Alsac, J. Bright, M. Prais and B. Stott, "Further Developments in LP-Based Optimal Power Flow," IEEE Transactions on Power Systems, 5(3), 1990, pp. 697711.

[11] Streiffert Dan. Multiple Area Economic Dispatch with line constrains IEEE Transactions on Power Systems, 1995,10(4), pp.1946-1951.

[12] Lee KY,Yome AS,Park JH "Adaptive Hopfield Neural Network For Economic Load Dispatch “IEEE Transactions on Power Systems, 1998,13(2),pp.519-526. 
[13] Song YH,Wang PV ,Johns AT. "Environmental Economic dispatch using fuzzy logic controller Genetic Algorithms "IEE Proc. Gener. Transm. Distr. 1997; 144(4), pp.377-382.

[14] F. Glover, "Tabu Search," University of Colorado, Boulder, CAAI Report 88-93, 1988

[15] S. Kirkpatrick, C. D. Gellat, and M. P. Vecchi, "Optimization by simulated annealing," Science, vol. 220, pp. 671-680, 1983.

[16] D. E. Goldberg," Genetic Algorithms in Search Optimization and Machine Learning "Addison-Wesley Publishing Company, Inc., 1989.

[17] Z.L. Gaing "Particle Swarm Optimization to Solving the Economic Dispatch Considering the Generator Constraints "IEEE Transactions on Power Systems, 2003,18(3), pp.1187-1195.

[18] S. Gerbex, R. Cherkaoui, and A. J. Germond, "Optimal Location Of Multi-Type FACTS Devices In A Power System By Means Of Genetic Algorithms," IEEE Transaction Power Systems, vol. 16, August. 2001, pp. 537-544.

[19] M.S.Osman, M.A.A-Sinna, A.A.Mousa "A Solution To The Optimal Power Flow Using Genetic Algorithm “MEPCON'2003 Power System, pp425-432.

[20] J. Kennedy and R. Eberhart, "Particle swarm optimization," Proc. IEEE Int. Conf. Neural Networks, vol. IV, pp. 1942-1948, 1995.

[21] P. Venkatesh, P. S. Kannan, and M. Sudhakaran, "Application of computational intelligence to economic load dispatch," J. Inst. Eng. (India), vol. 81, pp. 39-43, 2000. 